\title{
Rupture of the Anterolateral Papillary Muscle Caused by a High Lateral Branch Occlusion
}

\author{
Kayo Sugiyama $^{1^{*}}$, Masaki Kano², Satoshi Takahashi², Nobusato Koizumi², Hitoshi Ogino² \\ ${ }^{1}$ Department of Cardiac Surgery, Aichi Medical University Hospital, Nagakute, Japan \\ ${ }^{2}$ Department of Cardiovascular Surgery, Tokyo Medical University, Tokyo, Japan \\ Email: *kayotaro3@gmail.com
}

How to cite this paper: Sugiyama, K., Kano, M., Takahashi, S., Koizumi, N. and Ogino, H. (2018) Rupture of the Anterolateral Papillary Muscle Caused by a High Lateral Branch Occlusion. Open Journal of Thoracic Surgery, 8, 6-12.

https://doi.org/10.4236/ojts.2018.81002

Received: January 28, 2018

Accepted: March 11, 2018

Published: March 14, 2018

Copyright (ㅇ 2018 by authors and Scientific Research Publishing Inc. This work is licensed under the Creative Commons Attribution International License (CC BY 4.0).

http://creativecommons.org/licenses/by/4.0/

\begin{abstract}
Although necrosis of the left ventricular papillary muscles during acute myocardial infarction is common, rupture of the anterolateral papillary muscle due to occlusion of high lateral branch is rare. We present a rare case of anterolateral papillary muscle rupture caused by occlusion of a high lateral branch of the left coronary artery. Although the patient was in cardiogenic shock on admission, she was successfully treated by emergency mitral valve replacement after percutaneous catheter intervention and intra-aortic balloon pumping support. This case implies that the anterolateral papillary muscle can have a single blood supply and that it can be ruptured by an occlusion of a high lateral branch. Prompt diagnosis, immediate mechanical stabilization and aggressive surgical treatment are essential to save this group of patients.
\end{abstract}

\section{Keywords}

Acute Myocardial Infarction, Papillary Muscle Rupture, Acute Mitral Regurgitation

\section{Introduction}

Although necrosis of the papillary muscle (PM) potentially occurs due to acute myocardial infarction (AMI), rupture of the anterolateral PM is rare because of its dual blood supply. The anterolateral PM has a dual blood supply from a diagonal branch and an obtuse marginal branch of the left circumflex artery (LCx) and its single blood supply of high lateral branch is rare. We present a rare case of rupture of the anterolateral PM caused by total occlusion of a high lateral branch of the left coronary artery causing acute-onset severe mitral regurgitation (MR). 


\section{Case}

A 78-year-old woman was admitted for sudden onset of chest pain who was 150 $\mathrm{cm}$ in height and weighed $36 \mathrm{~kg}$. On admission, she presented with cardiogenic shock. Physical examination revealed the following: severe cardiogenic shock; blood pressure 51/32 $\mathrm{mmHg}$; heart rate 75 beats/min; no remarkable heart murmurs; crackles in both lungs; deteriorating consciousness. Electrocardiogram showed ST elevation in the I and aVL leads and ST depression in the II, III, aVF, and lateral chest leads. Echocardiography revealed akinesis of the left ventricle lateral wall and moderate mitral regurgitation (MR) caused by prolapse of the anterior mitral leaflet (Figure 1(a)). With the diagnosis of AMI, emergency coronary angiography was performed 30 minutes after the admission, which revealed total occlusion of a high lateral branch of left coronary artery and mild stenosis of the left anterior descending artery (Figure 1(b)). Following percutaneous catheter intervention (PCI) for total occlusion of the high lateral branch (Figure $1(\mathrm{c})$ ) was performed successfully 2 hours later after the onset, however, her conditions were rather deteriorating. Chest radiography after the PCI showed severe bilateral pulmonary edema without cardiomegaly (Figure 2(a)), and following echocardiography revealed massive MR with prolapse of the

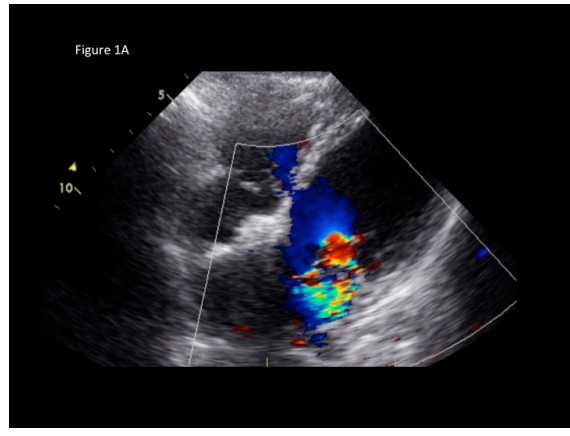

(a)

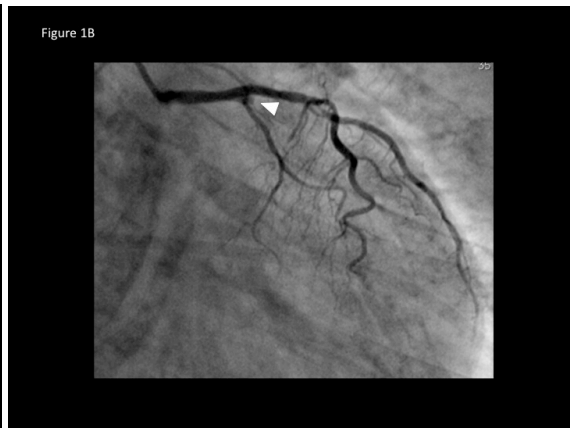

(b)

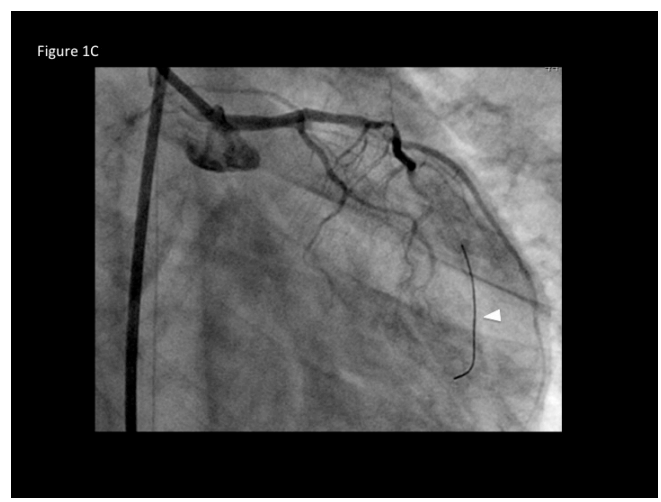

(c)

Figure 1. (a) Echocardiography revealed akinesis in the lateral wall and moderate mitral regurgitation caused by prolapse of the anterior mitral leaflet; (b) Emergency coronary angiography revealed an occlusion at the proximal site of the high lateral branch (arrowhead) and stenosis of left anterior descending artery (dotted arrow); (c) Percutaneous catheter intervention was performed to the high lateral branch (arrowhead). 


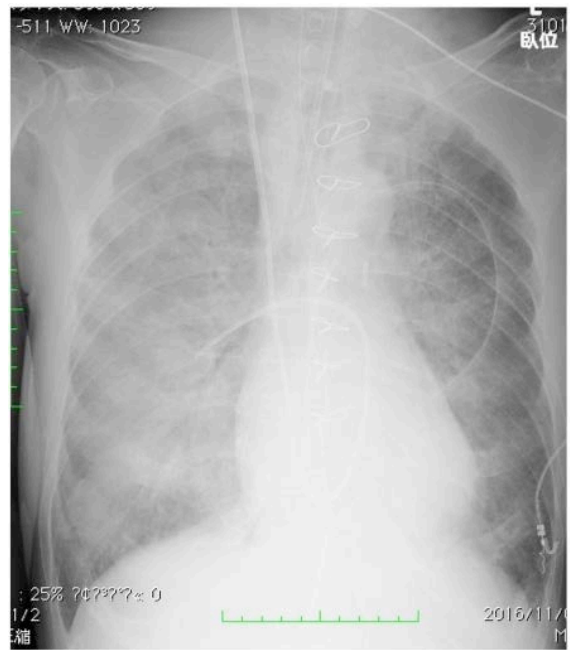

(a)

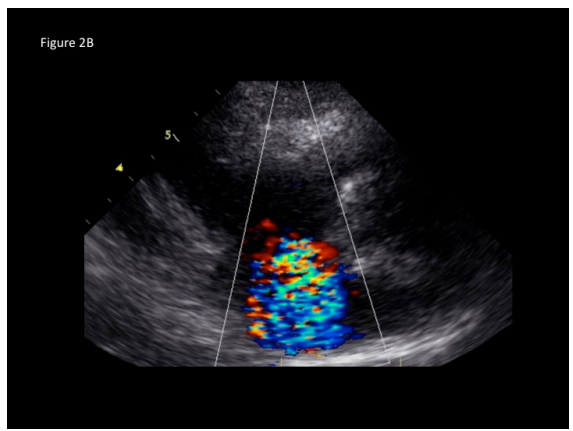

(b)

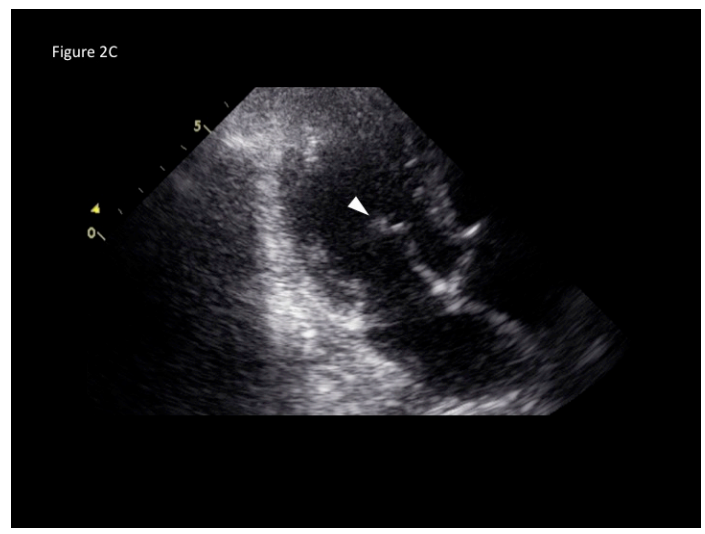

(c)

Figure 2. (a) Chest radiography showed acute pulmonary edema without cardiomegaly; (b) Echocardiography revealed massive mitral regurgitation; (c) Echocardiography revealed a ruptured anterior papillary muscle (arrowhead).

anterior mitral leaflet due to anterolateral PM rupture (Figure 2(b)). The ruptured anterolateral PM could be observed moving freely with the massive MR flow (Figure 2(c)). To improve the hemodynamic state, intra-aortic balloon pumping was also started in addition to revascularization.

\section{Surgery and Postoperative Course}

Immediately after the PCI, the patient was transferred to the operating room. Trans-esophageal echocardiography showed severe MR due to the ruptured anterolateral PM in the small left atrium. Complete rupture of the anterior head of anterolateral PM attached to the A1-2 part of mitral leaflet was found (Figure 3). In addition, some localized necrotic changes of the opposite site of ruptured edge of anterior PM were also recognized, but the other parts of left ventricle had no significant necrotic changes. Mitral valve replacement with preservation of the half of anterior mitral leaflet and the full of posterior mitral leaflet was performed using a $25 \mathrm{~mm}$ porcine bioprosthesis. Weaning from cardiopulmonary 


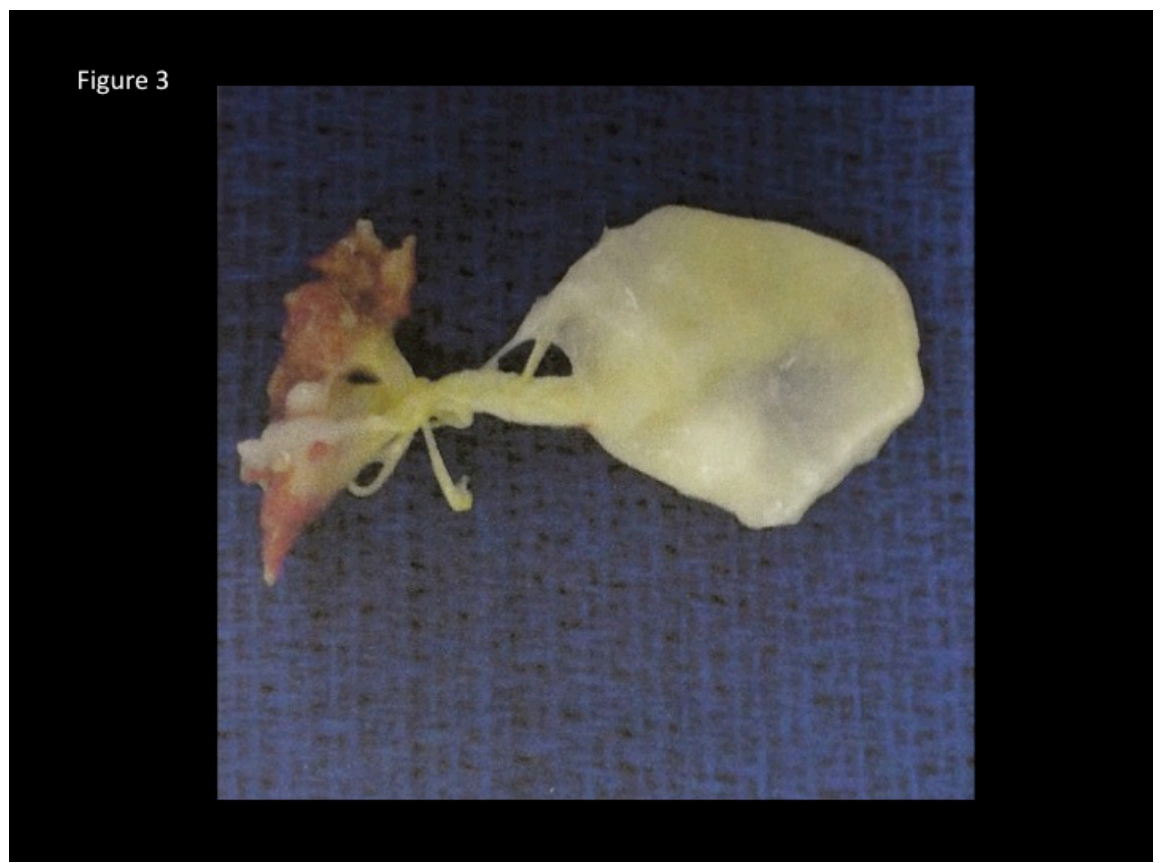

Figure 3. Resected specimen showed complete rupture of the anterior PM which showed necrotic degeneration, but chordae tendinae and mitral leaflet were relatively normal.

bypass with the support of IABP was smooth and the postoperative course was uneventful. The patient was discharged on the postoperative day 52 with the condition of New York Heart Association class I after the sufficient rehabilitation after AMI according to the patient's wish. The pathological findings of the resected anterior PM also showed diffuse myocardial necrosis due to AMI. She is currently doing well with no cardiac events at a follow-up of 15 months.

\section{Discussion}

Austen et al. reported the first case of successful mitral valve replacement in 1965 [1]. Fortunately, with an increasing emphasis on early coronary revascularization coupled with improved techniques, these catastrophic complications have become relatively rare, representing $2.3 \%$ of AMI [2]. Reportedly, $70 \%$ of patients with PM rupture developed cardiogenic shock, $70 \%$ of which died within 24 hours [3]. In another report, PM rupture almost always leaded to cardiogenic shock and carried the mortality at the incidence of $80 \%$ in the first week and $94 \%$ within 2 months [4].

The mechanisms are responsible for ischemic mitral valve prolapse have been linked to the anatomy of the individual PMs. According to Barbour and Roberts, the posteromedial PM, presumably because of its more tenuous blood supply, ruptured almost 3 times more frequently than the anterolateral PM $(73 \%$ and $27 \%$, respectively) [5]. The posteromedial PM has a single blood supply provided by the posterolateral artery from the right coronary artery or the left circumflex artery (LCx), and the anterolateral PM has a dual blood supply from a diagonal branch and an obtuse marginal branch of the LCx [6] [7] [8] [9]. 
Previous reports have found that rupture of the anterolateral PM followed an obstruction of the diagonal branch [6] [7] [10]. To the best of our knowledge, this is a rare report showing that a single obstruction of the high lateral branch caused an anterolateral PM rupture [11]. Moreover, the patient's anatomy of a hypoplastic LCx increased the possibility of PM rupture by an occlusion of only one high lateral branch as a morphological factor (Table 1). Furthermore, as patients' factors (Table 1), the present case was a small elderly female so that her tissues seem to be extremely fragile.

Some reports declared possibility of reperfusion injury because of delayed revascularization [12]. In the present case, reperfusion of the ischemic area after PCI might have related to the rupture of PM. Though PCI was performed only two hours later after the onset, injury of myocardium can happen if the ischemic area is large [12].

Treatment for this disease often necessitates emergent surgical intervention with mitral valve repair (if muscle necrosis is limited) or valve replacement [11]. Both immediate and long-term outcomes are improved with concomitant coronary revascularization [11]. The valve may be repaired in selected cases. The tissue is often weak and friable, making its repair difficult or impossible. In the present case, mitral valve replacement using tissue valve was chosen because of the complete anterolateral PM rupture and her advanced age. Early recognition, immediate medical stabilization, and aggressive treatment are essential [13].

In the present case, we observed a flailing mitral leaflet and extension of a portion of that leaflet into the left atrium during systole on transthoracic echocardiography, however, PM rupture was not detected on arrival. Transthoracic echocardiography is often the first imaging modality used in diagnosing PM rupture, with a sensitivity ranging from 65\% to $85 \%$ [14]. Transesophageal echocardiography is useful for distinguishing PM rupture if the transthoracic echocardiography images are poor [10].

We present a rare case of rupture of an anterolateral PM caused by total occlusion of the high lateral branch leading to severe MR. Although the incidence appears to be on the decline, clinicians must keep this lethal complication of AMI in mind when evaluating unstable patients. After the establishment of a definitive diagnosis, immediate medical and mechanical stabilization and aggressive surgical treatment are essential to save this group of patients.

Table 1. Sociodemographic and clinical characteristics of this patient.

\begin{tabular}{cc}
\hline Morphological factors & $\begin{array}{c}\text { Single blood supply } \\
\text { Hypoplastic circumflex artery }\end{array}$ \\
Patient's factors & Fragileness \\
& (elderly, female, low body weight) \\
& Delayed diagnosis
\end{tabular}




\section{Conflicts of Interest}

We have no disclosures to make and we did not receive any financial support for this study.

\section{References}

[1] Austen, W.G., Sanders, C.A., Averill, J.H. and Friedlich, A.L. (1965) Ruptured Papillary Muscle: Report of a Case with Successful Mitral Valve Replacement. Circulation, 32, 597-601. https://doi.org/10.1161/01.CIR.32.4.597

[2] Yuan, S.M., Jing, H. and Lavee, J. (2011) The Mechanical Complications of Acute Myocardial Infarction: Echocardiographic Visualizations. Turkish Journal of Thoracic and Cardiovascular Surgery, 19, 36-42.

[3] Wei, J.Y., Hutchins, G.M. and Bulkley, B.H. (1979) Papillary Muscle Rupture in Fatal Acute Myocardial Infarction: A Potentially Treatable form of Cardiogenic Shock. Annals of Internal Medicine, 90, 149-153.

https://doi.org/10.7326/0003-4819-90-2-149

[4] Sanders, R.J., Neubuerger, K.T. and Ravin, A. (1957) Rupture of Papillary Muscles: Occurrence of Rupture of the Posterior Muscle in Posteriod Myocardial Infarction. Diseases of the Chest, 31, 316. https://doi.org/10.1378/chest.31.3.316

[5] Barbour, D.J. and Roberts, W.C. (1986) Rupture of a Left Ventricular Papillary Muscle during Acute Myocardial Infarction: Analysis of 22 Necropsy Patients. Journal of the American College of Cardiology, 8, 558-565. https://doi.org/10.1016/S0735-1097(86)80182-6

[6] Wada, H., Yasu, T., Murata, S., Ohta, M., Kubo, N., Fujii, M., Kuroki, M., Kawakami, M. and Saito, M. (2002) Rupture of the Anterolateral Papillary Muscle Caused by a Single Diagonal Branch Obstruction. Circulation Journal, 66, 872-873. https://doi.org/10.1253/circj.66.872

[7] Kim, T.H., Seung, K.B., Kim, P.J., Baek, S.H., Chang, K.Y., Shin, W.S., Choi, K.B. and Moon, S.W. (2005) Anterolateral Papillary Muscle Rupture Complicated by the Obstruction of a Single Diagonal Branch. Circulation, 112, e269-e270. https://doi.org/10.1161/CIRCULATIONAHA.104.505016

[8] Voci, P., Biolotta, F. and Caretta, Q. (1995) Papillary Muscle Perfusion Pattern. A Hypothesis for Ischemic Papillary Muscle Dysfunction. Circulation, 91, 1714-1718. https://doi.org/10.1161/01.CIR.91.6.1714

[9] Nishimura, R.A., Gersh, B.J. and Schaff, H.V. (2000) The Case for an Aggressive Surgical Approach to Papillary Muscle Rupture Following Myocardial Infarction: "From Paradise Lost to Paradise Regained". Heart, 83, 611-613. https://doi.org/10.1136/heart.83.6.611

[10] Takahashi, T., Kohno, K., Kashida, M., Morita, T., Saito, K., Kamei, A., Seo, Y., Kawamura, I., Kojima, T., Seki, Y., Saito, K., Kumagai, K., Ohno, K., Tanaka, Y., Itaoka, Y., Okazaki, O., Izumo, K., Kimura, S., Akatsuka, N. and Yazaki, Y. (2002) A Survival Case of Acute Mitral Regurgitation and Cardiogenic Shock Caused by Subtotal Occlusion of the First Diagonal Branch. Circulation Journal, 66, 615-618. https://doi.org/10.1253/circj.66.615

[11] Abu Saleh, W.K., Aljabbari, O., Ramlawi, B. and Ramchandani, M. (2015) Necrosis of the Anterolateral Papillary Muscle-An Unusual Mechanical Complication of Myocardial Infarction. Debakey Journal, 11, 48-50.

https://doi.org/10.14797/mdcj-11-1-48 
[12] Yip, H., Wu, C., Chang, H., Wang, C., Cheng, C., Chua, S. and Chen, M. (2003) Cardiac Rupture Complicating Acute Myocardial Infarction in the Direct Percutaneous Coronary Intervention Reperfusion Era. Chest, 124, 454-571. https://doi.org/10.1378/chest.124.2.565

[13] Ma, H.-H., Honma, H., Munakata, K. and Hayakawa, H. (1997) Mitral Insufficiency as a Complication of Acute Myocardial Infarction and Left Ventricular Remodeling. Japanese Circulation Journal, 61, 912-920. https://doi.org/10.1253/jcj.61.912

[14] Czarnecki, A., Thakrar, A., Fang, T., Lytwyn, M., Ahmadle, R., Pascoe, E. and Jassal, D.S. (2008) Acute Severe Mitral Regurgitation: Consideration of Papillary Muscle Architecture. Cardiovascular Ultrasound, 6, 5.

https://doi.org/10.1186/1476-7120-6-5 